\title{
Succinct Review on MAC Protocols for Wireless Body Area Networks
}

\author{
Deepshikha* and Siddhartha Chauhan \\ Department of Computer Science \& Engineering, National Institute of \\ Technology, Hamirpur, H.P, 177005, India \\ deepshikha@live.in, siddharthachauhan1@gmail.com
}

\begin{abstract}
In the past decade, the growth of biomedical sensors has enabled a modernistic generation of wireless sensor networks viz. wireless body area networks (WBAN). These biomedical wireless sensor networks are equipped with tiny and low power body sensors which promise novel uses in healthcare, fitness and entertainment. Managing and controlling of Wireless Body Area Networks are complex and difficult tasks and efficient management of body sensor networks requires media access control protocols for power management. Different types of body sensors generate different types of data traffic, so WBAN requires MAC protocols for communication that are different from the traditional WSN MAC protocols. In this paper we have reviewed various MAC protocols of WBAN being used in healthcare monitoring applications. We have also classified them on the basis of channel access mechanism and baseline wireless standards.
\end{abstract}

Keywords: WBAN, architecture, biomedical sensors, MAC protocols

\section{Introduction}

Wireless Body Area Networks (WBAN) is an emerging low power technology that enables communication between body sensor nodes, (implanted or placed on human body) and a central coordinator. WBAN supports applications like remote patient monitoring in e-Health services [5]. Wireless sensor network is a key technology which has the potential to revolutionize health care services and can remarkably improve the health monitoring for patient care and reduce the cost associated with healthcare applications. In WBANs, the goal of the healthcare system is to assure the continuous and reliable gathering of physiological and behavioral aspects of the patients and deliver this information to medical care centers and physician. Each body area sensor networks consist of multiple inter-connected nodes placed on the human body or implanted inside the body, which together provide sensing, processing and communication capabilities. A WBAN can be seen as a physical layer for healthcare professional systems, collecting data to support healthcare professionals on their decisions and medical diagnosis.

A major requirement in wireless body area networks is low power consumption of system. Medium access control layer is well suited for achieving higher energy efficiency. MAC layer is also the core layer for achieving of quality of service $(\mathrm{QoS})$ provisioning [23]. For fulfilling the MAC requirements such as energy efficiency, scalability, QoS, reliability, bandwidth utilization various protocols have been proposed. In this paper we will provide a succinct review on various MAC protocols viz. energy efficient protocols, context aware protocols and QoS based protocols for wireless body area networks.

A survey on energy efficient MAC protocols for WBAN and a listing of various open challenges regarding medium access techniques have been provided in [23].

Received (November 10, 2017), Review Result (January 10, 2018), Accepted (January 20, 2018)

* Corresponding Author 
The power efficient protocols for body sensor networks were reviewed in [22], and the authors have also provided valuable suggestions for designing low power MAC protocols. Various proposed protocols based on the IEEE 802.15.6 [29] and IEEE 802.15.4 [30] were compared by [20]. Analysis of various QoS requirements of BAN done in [3], discussed about requisites of QoS handling system and compared previous works which were focuses on QoS provisioning in MAC layer. Comparison of different MAC protocols over wireless body area networks is provided in [32], where analysis of MAC protocols were carried out to find the best MAC protocol suitable of WBAN and focuses of performance enhancements of these networks. Few energy efficient protocols for WBAN have been reviewed in $[33,34]$ and some open challenge were also discussed. Several more MAC protocols have also been surveyed in [9], [24] \& [25]. We have reviewed MAC protocols which were proposed in the period 2008-2015 and have classified them on the basis of channel access mechanism and baseline wireless standard. The rest of the paper is organized as follows. Section II describes the architecture of Body Area Networks. Section III briefs about the MAC layers and associated challenges. Section IV gives the classification and Section V briefly explains several MAC protocols with their salient features followed comparison and conclusion.

\section{Brief Overview of Architecture of WBAN}

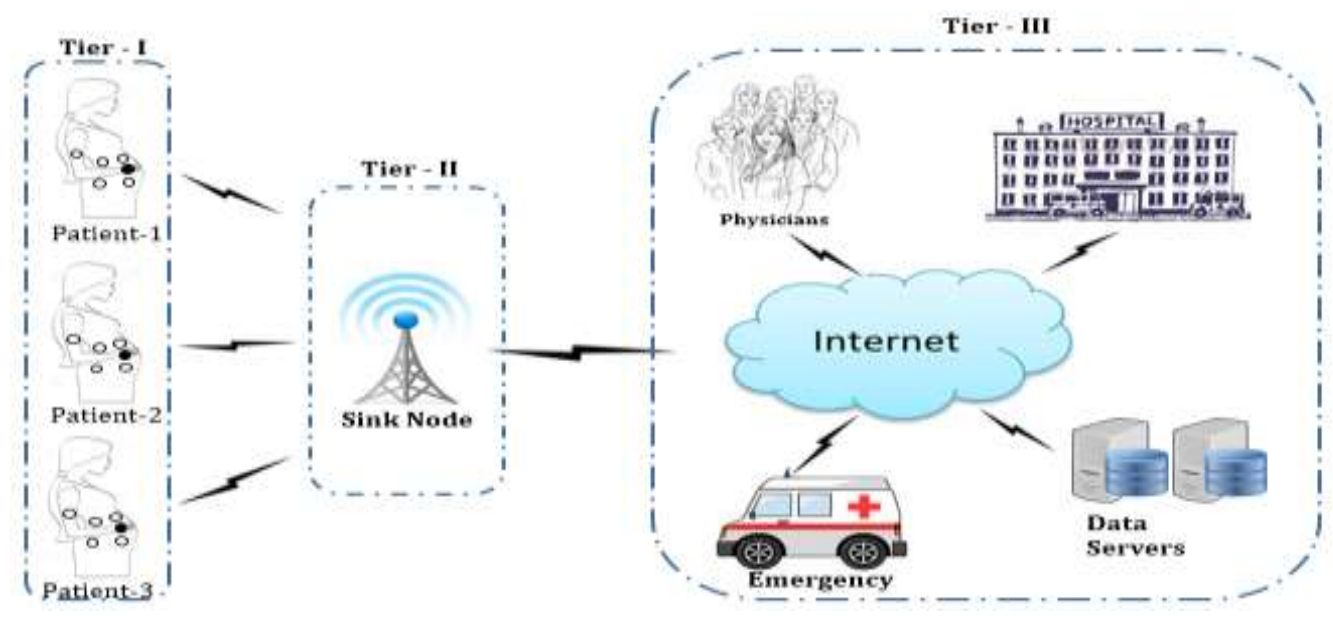

Figure 1. WBAN Architecture

WBAN is categorized mainly into two categories viz. flat architectures and multi-tier architectures [9]. In flat architectures each sensor node sends the data to personal server applications running on the PDA whereas in multitier architecture multiple sensor nodes in base tier collectively send the data to the PDA (at the third layer) through the gateway at second tier which acts as an interface between the base tier and the third tier as shown in Figure-1. The body sensor network generally has multi-tier architecture or three-stage architecture, in which each layer comprises of unique location and performs various functions [7]. First layer is comprised of multiple body sensors, data hub at the middle/interface tier and topmost stage/medical network of healthcare servers, caregivers or physicians, emergency services and medical researchers.

\section{MAC Layer and Associated Challenges}

At the medium access control (MAC) layer, the wireless short range communication network is formed between the data hub and the sensors. The wireless channel is shared among multiple sensor nodes and this access is controlled by MAC layer. In [8] the authors have done some implementations of WBAN using Bluetooth (802.15.4) for data 
transmission and observed that Bluetooth is high energy consuming and does not support multi-hop communication, so it is not suited for body area networks. Other enabling technologies used for WBAN implementations are IEEE 802.15.4 [30] \& IEEE 802.15.6 [29].

Challenges faced by WBAN at MAC are QoS provisioning to support the need of WBAN to achieve minimum acceptable limits on reliability, bandwidth and latency.

Energy efficiency: The trade-off analysis between the sensor node size and battery life depending upon the applications leads to energy scavenging. As the size requirements limit the battery size, power available from these batteries is small. Research is required to design pervasive scheduling techniques and power efficient scheme for achieving extreme energy efficiency [10].

Managing interference: An interference mitigation scheme is required to adapt faster than the network topology changes and helps in minimizing interference. When multiple people wearing body sensors come into the range of each other, then coordination between them become complex because it is difficult to predict their network's viewpoint.

High throughput: BAN needs to support higher range of throughput rates viz. $1 \mathrm{~kb} / \mathrm{s}-$ $10 \mathrm{Mb} / \mathrm{s}$ for high throughput applications such as video.

\section{Classification of MAC Protocols}

Generally, WBAN based MAC protocols are categorized as schedule-based and contention based. CSMA/CA is example of contention based whereas TDMA is a typical scheme of schedule based. In this paper we have classified the MAC protocols on basis of channel access mechanism viz. scheduled based, contention based, hybrid and wireless communication standards such as IEEE 802.15.4 [30] \& IEEE 802.15.6 [29]. A node is designated as coordinator in schedule based protocols to ensure the equal access of wireless medium among multiple senor nodes. The nodes do not compete for channel sharing because of proper time synchronization, so it is free from overhearing problem, packet loss due to collision and occurrence of idle listening.

In contention based protocols, all sensor nodes predetermine the access of channels using distributed channel sharing mechanism. The main advantages of contention based protocols are good adaptability to traffic variations.

The hybrid access mechanism uses both scheduled and contention approaches to achieve the requirement of applications thereby benefiting from advantages of both. The contention and scheduled mechanism are combined to support scalability and collision avoidance. WBAN protocols such as CA-MAC [5], P-MAC [20] \& PLA-MAC [12] have adopted this mechanism.

IEEE 802.15.4 [30]: This communication standard has been designed to support lowrate wireless personal area network (LR-WPAN) devices. This defines both physical and medium access layer. It operates in two modes: non-beacon mode and beacon enabled mode [21].

Non beacon Mode: This mode helps in full optimization of sensor nodes and accesses the channel only when it is necessary. The device can send data only if channel is free otherwise it has to wait for random period of time which is defined in the standard. The coordinator has to wait for data request from the sensor device for sending data to that node and after sending acknowledgement of request, data can be transmitted to device by using CSMA/CA technique.

Beacon enabled Mode: beacon is used to synchronize all the devices with coordinator by operating periodically. In this mode, the coordinator has an active period and inactive period to save the energy. This period of active part and inactive part is known as super frame structure. The active period of super frame structure has 16 time slots which are further divided into contention access period (CAP) and contention free period (CFP). 
Where CAP is similar to beaconless mode where any device can transmit randomly within the time slots. And CFP provide guaranteed channel access to a device through specific number of slots called guaranteed time slots (GTS).

IEEE 802.15.6 [29]: This standard has been designed for supporting wireless body area networks which operate in and around the human body. This operates on very low frequency (less than $1 \mathrm{MHz}$ ) for reliable wireless communication. The super frame structure of IEEE 802.15.6 supports nine access phases which are beacon, beacon 2, two exclusive access phases (EAP1 \& EAP2), two random access phases (RAP1 \& RAP2), two managed access phases (MAP1 \& MAP2) and Contention access phase (CAP) as shown in Figure 2 [29].

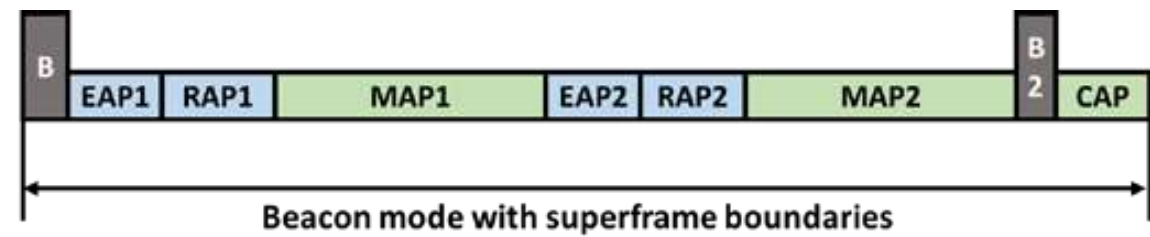

Figure 2. Access Phases of a Super Frame in Beacon Mode IEEE 802.15.6

The beacon initializes the super frame, EAP1 \& EAP2 are used for emergency traffic, RAP1, RAP2 \& CAP phases are dedicated for normal traffic, MAP is responsible for managing uplink, downlink and bi-link allocation intervals and beacon 2 frame indicates the starting and end point of CAP. We have further classified the reviewed protocols, as shown in Figure 3, on the basis of energy efficiency, data prioritization and QoS support.

Energy efficient protocols: Energy aware communication algorithms are required to enhance the performance of the networks. Energy efficiency can be incremented by minimizing the energy wastes like packet collision, idle-listening, overhearing, overemitting and traffic fluctuations.

Data-prioritization protocols: Data-prioritization algorithms are those wherein collected/sensed data is classified into sub levels so that emergency medical data can be transmitted in the expected time.

QoS supportive algorithms: QoS (Quality of service) is defined as guarantee of level of service provided by the network. The protocols which can set minimum acceptable limits on reliability, latency and bandwidth are able to provide QoS provisioning to the network.

\section{MAC Protocols for WBAN}

$\boldsymbol{H}$-MAC [15] (Heart-Beat Driven MAC protocol) is schedule based TDMA protocol which uses heartbeat rhythm information for time synchronization to improve energy efficiency, instead of using periodic beacons for synchronization. The heartbeat rhythm generated by rhythmicity of heart muscles of person and most of bio-signals have same rhythms, and changes in the rhythms are detected by bio-sensory signals.

Features:

- The sensory data or bio-signals gathered by sensors are represented by peak sequences of waveforms, peak detection algorithms are applied to collected peak sequences for extracting required information for node synchronization. In this way the coordinator gets timing synchronization information without turning on node's radios and saving energy.

- For time slot scheduling in H-MAC, peaks are used as synchronization beacon and peak interval used as data transmission slots. 
- The coordinator is responsible for control messages for synchronization or resynchronization and time slot scheduling. For synchronization control packet CS packet are used which include coordinator's current peak counting number and one bit for checking whether there is any change in slot assignment. After using CS packet CL packets are used for slot scheduling which include frame length, start/stop of peak number, sensor ID and mandatory radio cycles. On receiving control packets all nodes can transmit their data in assigned peak intervals and start their respective peak counters.

- Resynchronization recovery scheme triggered if sensor node predicts a synchronization loss. This synchronization scheme is based on two predefined threshold values T1 \& T2. T1 is represented by samples between current peak interval and average previous peak interval. If an abrupt change in peak interval detected then it is compared with second predefined threshold value $\mathrm{T} 2$, if value exceeds $\mathrm{T} 2$ then synchronization recovery scheme is activated.

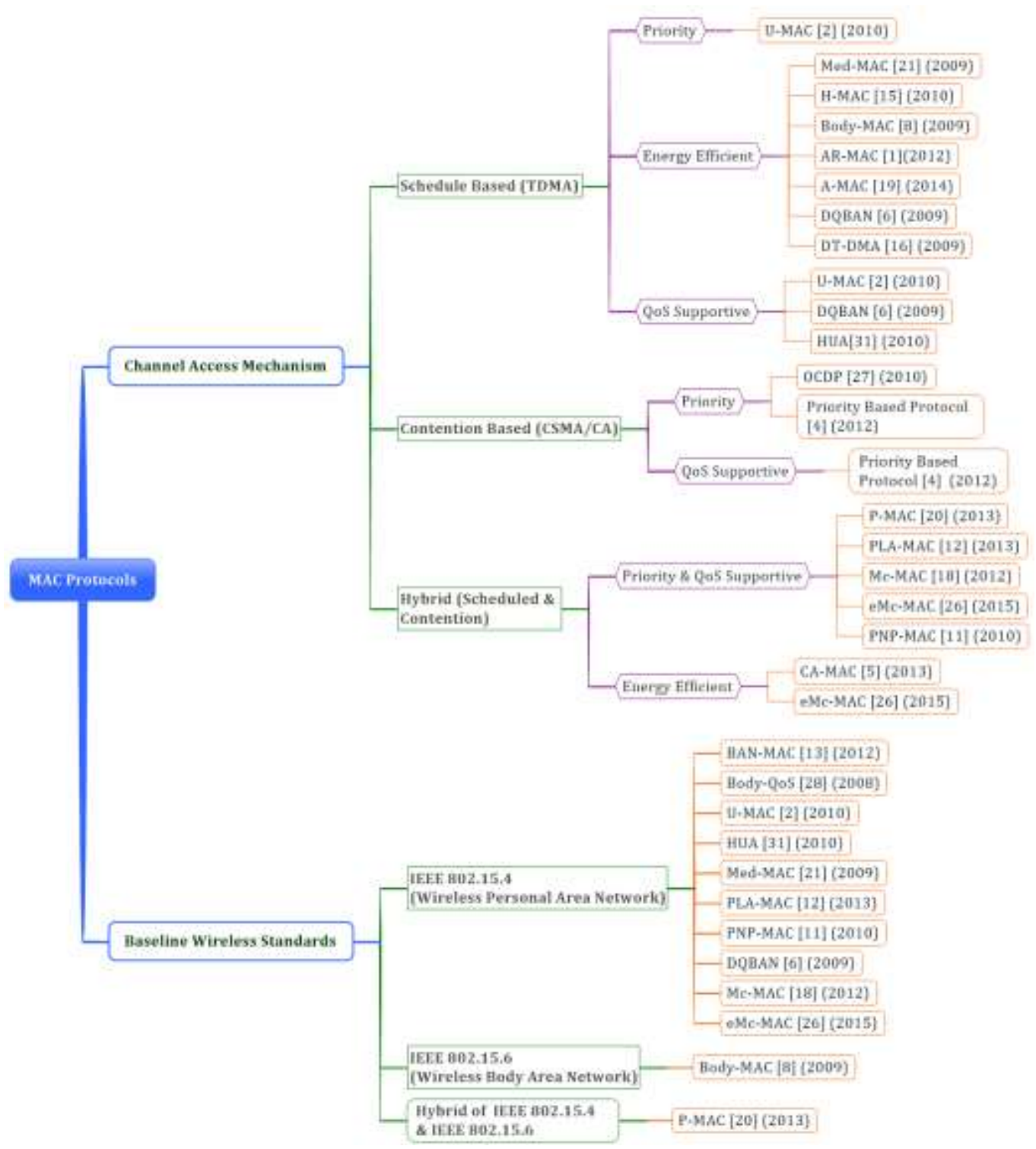

Figure 3. MAC Protocol Classification 
- H-MAC protocol minimizes extra energy required for synchronization. H-MAC protocol consider the time slot scheduling for one hop star topology and perform better in this topology.

$\boldsymbol{U}$-MAC (Urgency MAC) [2] is a priority driven MAC protocol targeted for medical applications. It is based on IEEE 802.15.4a standard.

\section{Features:}

- In U-MAC health information is classified into priority levels on the basis of traffic such as critical/higher priority information or non-critical/low priority information which is denoted by traffic classes $\mathrm{C} 1 \& \mathrm{C} 2$, and maximum number of allowed retransmissions are also defined viz. $\mathrm{r} 1 \& \mathrm{r} 2$ respectively

- In Urgency-based MAC protocol, slotted Aloha access mechanism is assumed in which sensor nodes compete for the medium only at the starting of each time slot of the MAC frame.

- Sensor node transmits the higher priority heath information by reducing the number of retransmissions of non-prioritized or low priority data. U-MAC helps in supporting quality of service provisioning in healthcare applications.

Body-MAC [8] is TDMA based protocol which is designed to be energy efficient and supportive to flexible bandwidth approaches in MAC frame structure. Energy efficiency of node is improved by reducing the packet collision; by avoiding idle listening and lowering the control packet overhead.

Frame structure: The Body-MAC frame structure consists of three parts viz. beacon, uplink and downlink sub-frames. The downlink portion is reserved for transmission from gateway to the nodes either in unicast or broadcast manner. And uplink portion is divided into contention Access period (CAP) and contention Free Period (CFP), where in CAP nodes compete for transmission of control or data packets and allocation of granted time slots for nodes is managed in CFP. The configuration of downlink and uplink \& uplink sub-frames can be flexibly changed according to traffic conditions.

Bandwidth management in uplink sub frame is done by making control and data bandwidth requests.

- For data transmission, data bandwidth request are made in burst, periodic and adjust bandwidth manner. Where burst bandwidth is used for temporary period of bandwidth and can be reused after some time in MAC frame, periodic bandwidth allow exclusively access to channel for few MAC frames and adjust bandwidth defines the amount of bandwidth to be added or reduced from the previous periodic bandwidth used by node.

- To avoid the wastage of energy, when nodes have to stay awake to receive data, if no data is available to receive, sleep mode is used and idle listening is avoided. Before going into sleep mode, the nodes perform three tasks: sleep mode request, sleep mode grant and sleep mode wakeup.

- At the beginning of sleep mode process node makes sleep mode request to the gateway including sleep duration in terms of frames, ACK sent from gateway implies that sleep mode has been granted.

- The sleep mode is specifically efficient for nodes which have support for low duty cycle applications. Body MAC offers better performance in terms of end to end delay.

Med-MAC [21] is energy efficient, adaptive and low overhead TDMA synchronization scheme based protocol which provide exclusively contention free channel access over variable channels. It can dynamically adjust the time slots and QoS requirements 
according to network traffic. It manages the device synchronization without waking the nodes for regular beacons.

Frame structure: Med-MAC uses an adaptive superframe structure combining the contention and dynamic slot reservation sections to support QoS traffic classes. These classes support:

- Class 0: low grade data (data rate <1000bps)

- Class 1: medium grade data (data rate $<=250 \mathrm{kbps}$ ) and,

- Class 2: high grade data (data rate <10Mbps).

For incorporating all three classes a multi-super frame structure is used which has dynamic and programmable sub frame unit bounded by beacon period. This beacon period include optional contention period and contention free period ranging from 2 to 256 timeslots. Duration of superframe and time slots are adjustable according to sleep/power saving demands.

Synchronization: Med-MAC introduces a new mechanism for synchronization among the nodes, wherein node can sleep through number of beacon periods without losing synchronization.

- It introduces guard or sleep mode mechanism which turn off node's radio during uplink, downlink and beacon periods as much as possible and helps in avoiding overlaps.

- The adaptive guard band algorithm supports the node to sleep through multiple beacons and uses guard band to calculate the actual drift between the time slots. This drift adjustment factor helps in minimizing the bandwidth waste.

- To achieve synchronization between coordinator and other nodes the combination of Adaptive Guard Band Algorithm (AGBA) and time stamp scavenging is used.

$\boldsymbol{A R}-\boldsymbol{M A C}$ [1] (Adaptive reliable medium access control protocol) is on TDMA based approach for minimizing energy consumption. Every sensor node is assigned with Guaranteed Time Slots (GTS) for communication to overcome the problems of packet collision and overhearing. AR-MAC allocates total time frame $\mathrm{T}^{\text {frame }}$ for communication to the sensor nodes. $\mathrm{T}^{\text {frame }}$ consists of three parts viz. contention free period (CFP), contention access period (CAP) and $\mathrm{T}^{\mathrm{MS}}$. These parts of total time frame are used for communication with sensors, on-demand traffic and for communication with master station (MS).

Channel Selection: For Channel Selection in AR-MAC protocol the coordinator node scans for free radio frequency. If channel is busy, the coordinator node switches off the current radio channel and switches on another radio channel until a free radio channel is found.

Time slot assignment: After channel selection sensor node sends TSR (time slot request) packet to the coordinator containing node's data rate and required time slot information. Coordinator node assigns the time slots on basis of node's traffic patterns and sends TSRR (Time slot request reply) packet to the requesting sensor node. Guard band time slots are inserted between two successive time slots to avoid the interference between different data rate and sampling intervals of the sensors.

Synchronization: Coordinator node listens for data packets in expected time slots after the time slot allocation. On arrival of data packet, coordinator node gets Drift Value (DV) by comparing the packet's current arrival time, expected arrival time with acceptable delay (D). If DV > D then coordinator node sends SYNC-ACK packet to sensor nodes for future synchronization otherwise sends simple Ack packet for received data packet. This drift value helps the sensor nodes to adjust their wake up time schedule for future communication.

$\boldsymbol{A}$-MAC [19] is adaptive medium control access protocol which is an enhancement of previously discussed AR-MAC protocol. It is also based on TDMA approach and uses 
adaptive guard band and adaptive time slot allocation as used in AR-MAC. In addition to that A-MAC focuses on the minimization of energy consumption of sensor node by limiting the number of channel access tries.

$\boldsymbol{D Q B A N}[6]$ is highly reliable MAC protocol which uses distributed queues as used in DQRAP (Distributed queuing random access protocol) for local wireless communication. DQRAP divide the TDMA slots into access sub-slots which are further divided into minislots and data subslots. Collision resolution queue (CRQ) and data transmission queue (DTQ) are used for controlling user access requests in minislot section and collision free data transmission in data subslot.

DQRAP uses first come first serve mechanism in DTQ whereas in DQBAN cross layer fuzzy rule based scheduler is used. This scheduler does not allow occupying first position in DTQ but it allows data transmission in next collision free frame.

$D Q B A N$ superframe structure: Frame structure is divided in two parts: one for communication from sensor nodes to BAN coordinator and other is BAN coordinator to sensor nodes. In body sensors to coordinator slots are divided into "m access minislots", "n scheduling minislots", and "data slots" which require $\mathrm{T}_{\text {access }}$ duration for accessing requests, $\mathrm{T}_{\text {scheduling }}$ for scheduling $\mathrm{n}$ minislots and $\mathrm{T}_{\text {Data }}$ duration of variable length for data transmission. Similarly, the BAN coordinator communicates to sensors by an ACK of duration $\mathrm{T}_{\mathrm{ACK}}$ to acknowledge the packet, synchronization preamble (pre) of duration $\mathrm{T}_{\text {pre }}$ for permitting energy-aware radio activation policies, and $\mathrm{T}_{\mathrm{FBP}}$ of fixed duration for broadcasting by BAN coordinator.

DQBAN is adaptive to traffic load, it behaves as slotted ALOHA for light traffic load and a reservation protocol for high traffic data. For achieving QoS requirements, it behaves as polling protocol to providing collision free data frames.

Body-QoS MAC [28] protocol implemented for QoS support in body area sensor networks for providing support to adapt heterogeneous traffic, reliable data communication and adaptive resource scheduling. Body-QoS MAC follows the asymmetric architecture to providing adaptive resource scheduling by using three components viz. admission control, QoS scheduler and virtual MAC.

The admission control and QoS scheduler act in master-slave manner on the aggregator and sensor nodes. Admission control is responsible for managing new QoS reservations and runtime estimation of the effective bandwidth. The acceptance and rejection to new QoS reservation depends upon constraints like required bandwidth, delay, priority, available wireless resources, estimated channel bandwidth and previous reservations.

Three types of traffic are supported by protocol which are aggregator to mote, mote to aggregator and best effort. Firstly, the bandwidth is assigned to aggregator to mote traffic in every transmission period. Then polling packets are generated for mote to aggregator traffic having information of number of data packets to be sent and granted time interval. In response of polling packet, data packets are sent by the scheduled nodes. After each cycle aggregator measures effective bandwidth by analyzing assigned time interval and number of data packets assigned and received. For determining number of data packets and time interval, Resource Reservation Light (RSVP-Light) QoS Scheduling and adaptive QoS scheduling techniques are used.

In RSVP-Light QoS scheme it is assumed that audio/video stream require fixed bandwidth reservation without retransmission provision. Body-QoS support this scheduling by reserving fixed bandwidth according to QoS requirements and ignoring the channel conditions and measured effective bandwidth. Adaptive QoS scheduling copes with the channel impairments by managing wireless resources adaptably.

$\boldsymbol{B A N}-\boldsymbol{M A C}$ [13] is an opportunistic MAC protocol for reliable communication in body area networks which is based on 802.15.4 ZigBee specifications. On the basis of received signal strength (RSS), BANMAC protocol monitors and predicts the channel fluctuations and schedule transmission opportunistically using opportune transmission window (OTW), when RSS is higher. RSS fluctuations are high when nodes are close to line of 
sight and weaker when they are in in non-line of sight. The RSS fluctuations in Body Area network on walking persons in various hardware platforms is denoted by Received signal strength indicator (RSSI) in [14]. For the future prediction of RSS fluctuations, BANMAC uses RSSI-based OTW prediction algorithm. This prediction algorithm works in three steps of a single cycle. In first step, the coordinator node collects the RSSI time series by periodically broadcasting RSSI probe packets at low frequency. Nodes record and store the RSSI value of these probe packets, then set of RSSI values and probe identifiers are sent back to the coordinator node. In second step, the coordinator node processes the collected RSSI time series to determine frequency fluctuations and in last step algorithm predicts OTWs using frequency and phase information.

BANMAC offers collision and conflict free channel access by pull based scheduling, where data transmissions are scheduled as of random approach. This protocol schedules the transmission on the basis of node's mobility. If node is on a mobile limb then it predicts the center of OTWs which lead to reliable communication of the protocol. And transmission from the stationary nodes is scheduled in remaining available time. BANMAC protocol provides support for both centralized and fully distributed coordination system.

DT-DMA [16] A Reservation-based Dynamic TDMA Protocol is developed for normal periodic WBAN traffic. In this protocol slots are allocated to the nodes which have buffered packets and these are released when data transmission completed. The channel is bounded by super-frame structures where every super-frame consists of a beacon that used to carry control information including slot allocation information. A configurable CFP period which is used for data transmission through allotted slots and a CAP period where short command packets are transmitted using ALOHA protocol. For earlier transmission of CFP traffic, CFP period comes before the CAP period unlikely in the frame structure of IEEE 802.15.4. After CAP period there is configurable inactive period i.e. if CFP traffic is minimal then inactive period will be increased. DT-DMA does not support emergency traffic and on-demand traffic and it shows more dependability for low packet dropping rate and low energy consumption as of IEEE 802.15.4.

$\boldsymbol{C A}-\boldsymbol{M A C}[5]$ is context-aware protocol designed to meet the time varying requirements of Body Area networks. CA-MAC is able to reduce latency, packet loss and energy utilization by applying traffic aware \& channel aware adjustment of transmission priority and channel access mechanism. This results in trade-off between efficiency and reliability of the network.

Frame-Structure: New hybrid super frame structure is used in CA-MAC, which is consisted on three parts beacon, contention and TDMA. Time duration of beacon and super frame remain constant while duration of TDMA or contention can be changed adaptively.

In the first super frame, the coordinator node broadcasts beacon packet to all nodes for synchronization. If there are any changes in traffic and channel conditions of the network then beacon packets are broadcasted to specify new super frame accordingly, otherwise super frame remains unchanged. Second part of super frame is further divided into two parts scheduled based slots and polling based slots. Scheduled based slots are dynamically reserved for nodes based on associated traffic conditions while polling slots are only assigned on demand to particular node otherwise remain inactive with no transmission. Traffic aware adjustment of transmission priority: CA-MAC dynamically changes the sampling rates for normal traffic and emergency traffic accordingly.

Under Normal context, the coordinator node receives the data from other nodes through its scheduled slots. All nodes go into sleep state, after data transmission is complete and sender node's buffer is empty. The super frame structure remains unchanged if coordinator node does not broadcast any new beacon packet. Emergency context is triggered if nodes receives newly broadcasted beacon packet which means there were some abnormalities during previous superframe and next superframe structure is updated accordingly. 
Emergency context is continued until coordinator broadcast a new beacon packet indicating that network is in normal context.

Channel aware adjustment of access Mechanism: To deal with channel fading contention based design is used. In order to set up contention operations appropriately, contention length index (CLI) is used which is ratio of NCDP \& TDC, where NCDP is number of consequently dropping packets and TDC is total times of data collision.

Initially in the starting of superframe structure, the duration of contention period is set to a low value and coordinator node calculates each node's NCDP and network's TDC by analyzing data transmission of the system. On the basis of node's average contention length index (ACLI) coordinator node calculates the final context length (FCL) of the next superframe. This FCL help in predicting channel conditions and enhances the transmission reliability.

$\boldsymbol{P}$-MAC [20]: A new priority MAC protocol has been designed to overcome the limitations such as reducing control overhead, avoiding idle listening and minimizing packet collision ratio. In this priority protocol whole network is controlled by the coordinator and it can choose the boundaries of superframe which are indicated by beacon. In PMAC data channels are separated from the control channels and priority is given to the emergency traffic.

Frame-Structure: PMAC uses hybrid frame structure containing downlink subframe (DL) and uplink subframe (UL). Downlink subframe starts with a frame dedicated for synchronization and equalization. Whereas uplink subframe is divided into two parts: TDMA and CAP, TDMA is further divided into timeslots reserved for normal traffic (TSRN) and emergency traffic (TSRE). In CAP contention based allocations are based on the CSMA/CA. CAP is partitioned into two control channels: in first part UCE is responsible for uplink control of emergency medical traffic and in second part UCN is responsible for controlling channel of normal traffic. PMAC has used two inner frame spaces called FIFS (emergency IFS) and NIFS (normal IFS). Every sensor node maintains a contention window $(\mathrm{CW})$ which maintains the times a sensor has to wait before transmission. An emergency node has to wait EIFS with EIFS <NIFS before transmitting packet and normal traffic has to wait NIFS before data transmission in UCN channels.

For energy saving sleep mode is used in PMAC. In this mode node send sleep request (SLP-REQ) to the coordinator for entering in sleep mode. The coordinator node sends SLP-RP in response of node's request if there is no data to transmit.

PLA-MAC [12]: It is traffic priority and load adaptive protocol for QoS provisioning in BSNs. In PLA-MAC sensed data packets are classified according to their QoS requirements and their priorities are calculated accordingly which helps in scheduling packet transmission based on priority. Data packets are categorized in four classes based on data type and their rate generation which are ordinary data packets (OPs), delay-driven data packets (DPs), reliability driven data packets (RPs), and critical data packets (CPs). These data packets are assigned with a data type number $T_{i}$, where critical data packets are assigned with lowest and ordinary data packets with highest data type number. This will helpful in calculation of back-off and priority values.

Frame Structure: PLA-MAC has used modified version of IEEE 802.15.4, which is dynamic superframe structure depends upon the variation of traffic load. The superframe structure is divided into five periods: beacon, contention access period (CAP), notification, Contention free period (CFP), and inactive period (IP). Each superframe starts with beacon, informing all members about basic information about coordinator and other nodes. In CAP, allocation request for CFP slots are sent for data packets of classes CPs, RPs and OPs. Data packets of class DPs are received by coordinator from the sensor nodes. After CAP, the coordinator allocates the data transfer slots (DTS) to packets and notifies to all nodes through notification. Higher priority packets are allocated first. The size of CFP is dynamic, which depends upon traffic load i.e. CFP is small when traffic load is low and CFP is larger by occupying the IP when load is higher. If CFP does not 
occupy the entire superframe then, inactive period is used for low power listening (LPL). In LPL, the sensor nodes can transmit emergency packets only, depending upon the implementations.

OCDP (Opportunistic Contention Decision period) [27]: OCDP is designed to support WBAN services for both medical applications and consumer electronics (CE). This protocol supports a temporary switching method through OCDP between the inactive period and opportunity period. To support communication in mesh topology for bursty CE data and emergency medical data, 4 mode opportunity periods are used.

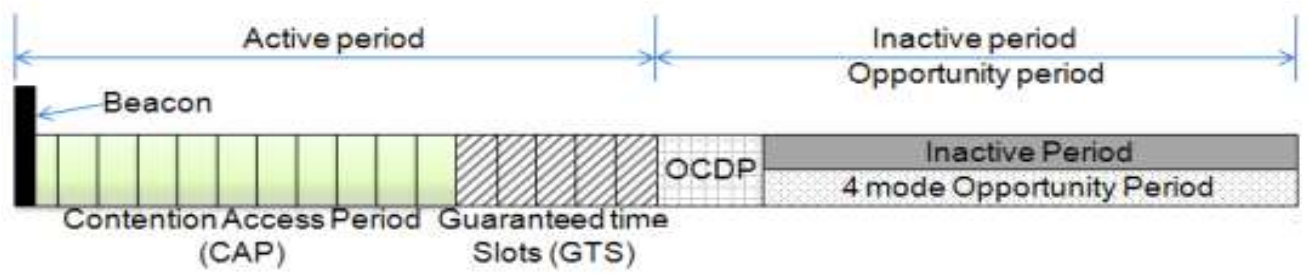

Figure 4. Time Slot Assignment

Frame-structure: Hybrid superframe structure is used to support WBAN devices required latency and heterogeneous traffic. OCDP (opportunistic contention decision period) provides an opportunity for temporary switching between the inactive period and opportunity period as shown in Figure $4 \& 5$ [27]. In this opportunity period 4 modes of opportunity periods are used. OCDP is composed of two sub periods RO (Request of opportunity) and AO (Acknowledgement of opportunity). OCM (opportunity contention message) and OCM ACK (OCM acknowledgement) request frames are used by sensor nodes to make requests to the coordinator node for opportunity period. Where RO1 OCM is used by WBAN devices or sensor nodes, $\mathrm{RO} 2$ used by consumer electronics (CE) with slotted CSMA/CA and RO3 are used by CE with CSMA/CA.

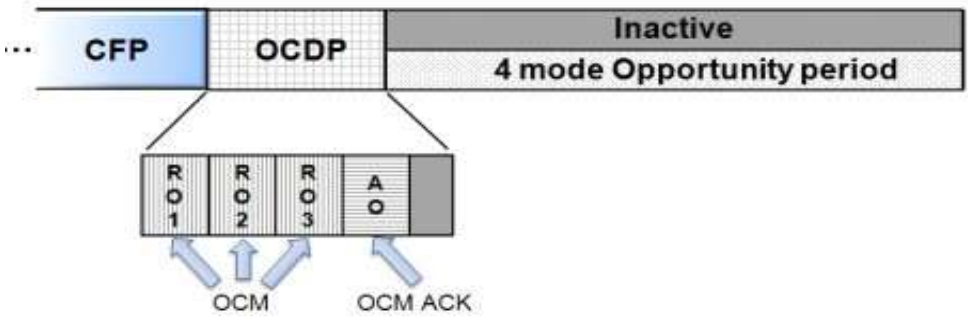

Figure 5. Detailed OCDP

Priority based channel Access algorithm for contention based MAC protocol [4]: This protocol classifies the contention based channel access phase into 4-levels of priority packets on the basis of priority which is defined in the WBAN draft document. Priority of packets are categorized on the basis of services like highest priority medical services, mixed medical and non-medical, non-medical and general health services. This classification helps the coordinator node to calculate average delay of every superframe.

In WBAN, TRD (technical requirement document) is used to define duty cycle, delay requirements for both medical and CE services. These delay requirements helps in defining delay threshold values for all levels of categories and divide the CAP into subphases. Categories 1,2 and 3 support medical traffic having delay threshold of $125 \mathrm{~ms}$, whereas category 4 supports non-medical traffic with delay threshold of $250 \mathrm{~ms}$. This protocol is more efficient in energy consumption and high channel utilization than baseline MAC 802.15.6. 
TAD-MAC: Traffic Aware Dynamic MAC Protocol for Wireless Body Area Sensor networks [17]: This protocol follows the dynamic adaptation of wake up interval of every node based upon traffic status register bank. It targets on both on invasive and non-invasive body area networks and follow hybrid network topology. It has considered star topology for in-body networks which means all nodes directly communicate with coordinator node and maintains TSR-Bank (traffic status register bank) for all transmitting nodes. TSR-bank contains the traffic statistics based on wake up intervals (WUInt) of receiving node with respect to data transmission rate of transmitting nodes. In case of non-invasive networks mesh topology is followed and all the nodes contain TSRbank of their neighboring nodes. This protocol falls into the category of preamble MAC protocols which do not require any scheduling mechanism and synchronization among the nodes. Every node is independent for its sleeping and periods. Communication can be started either by transmit node or receiver node. In this protocol communication is started by receive node. It is divided into two phases: evolution phase and steady phase. Before convergence or in evolution phase coordinator node collects gather wake-up interval patterns by analyzing several wake up interval of other transmit nodes. This WUInt is adapted by coordinator in steady phase in such a way so that idle listening is minimized.

McMAC [18]: This protocol is designed to provide multi-constrained QoS provisioning for wireless body area networks. It has proposed a new superframe structure which works on the "transmit whenever appropriate" mechanism and allow separate transmission periods for different traffic classes. Traffic is divided into five traffic classes where type 0 corresponds to emergency data, type 1 is both delay and reliability constrained, type 2 is reliability constrained but not delay, type 3 is delay constrained only and type 4 is neither delay nor reliability constrained. For type 0 traffic type a new mechanism is introduced which is based on poll based data transmission and results in high reliability and least possible delay.

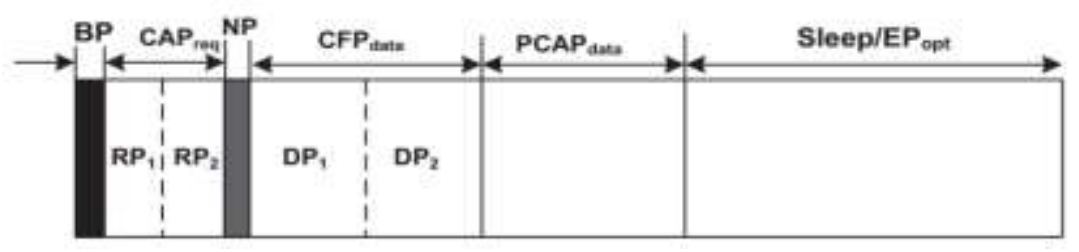

Figure 6. Super Frame Structure of Mc-MAC

Superframe structure: This superframe structure uses a hybrid frame structure which follows both contentions based and contention free approach. It has different periods for diverse traffic classes according to QoS requirements. Frame structure starts with beacon period (BP), followed by $\mathrm{CAP}_{\text {req }}, \mathrm{NP}, \mathrm{CFP}_{\text {data }}, \mathrm{PCAP}_{\text {data }}$ and Sleep/ emergency period optional as shown in Figure 6 [18]. In beacon period coordinator broadcasts beacon to other nodes and in $\mathrm{CAP}_{\text {req }}$ nodes having type 1 and type 2 traffic send request in Request

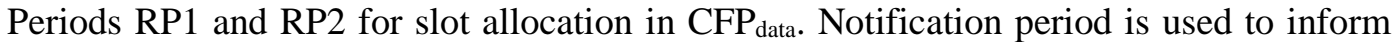
about allocated slots from coordinator to transmitting nodes. After NP, $\mathrm{CFP}_{\text {data }}$ starts, where type 1 and type 2 data is transmitted through Distinct Periods DP1 and DP2 previous allocated slots in $\mathrm{CAP}_{\text {req. }}$ For both type 3 and type 4 traffic type, there is prioritized contention access period $\left(\mathrm{PCAP}_{\text {data }}\right)$ and last part is sleep or optional emergency period where nodes remain in sleep state or node having type 0 data can transmit the data.

eMcMAC [26] : It is a low-overhead multi-constrained QoS aware MAC protocol which is designed in such a way so that critical packets can be transmitted earlier than other packets. The concept of minislots is introduced in CFP for immediate transmission on urgent packets. In this protocol sensed data traffic is also divided into five levels viz. urgent packets, critical packets, reliability constrained packets, delay constrained packets 
and normal packets. A higher priority data packet gets a better chance to request for GTS instead of lower priority packets by using prioritized random back off.

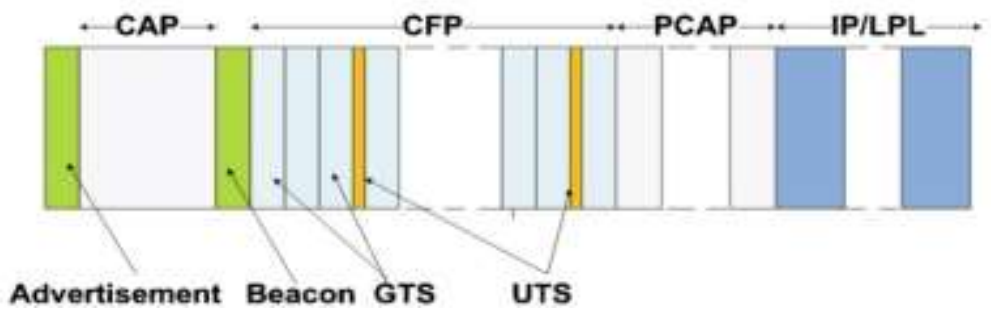

Figure 7. Super Frame Structure of eMc-MAC

Superframe structure: eMC-MAC has a flexible superframe structure to support the traffic adjustments according to application's requirements. Frame structure starts with advertisement in which coordinator broadcast the network information and synchronizes with other BSN nodes. In CAP, critical and reliability constrained packets make GTS requests. Through beacon period coordinator informs other nodes about slot allocations in CFP. Mini slots called UTS (urgent time slots) are kept on regular interval in CFP for transmission on urgent packets. These slots are kept on regular interval of time and much smaller than regular GTS slot. For transmission of delay constrained and normal packets there is PCAP period followed by inactive period where nodes go into sleep state or low power listening state. Detailed frame structure is listed in the Figure 7 [26].

\section{Comparison of MAC Protocols}

Comparison of above discussed protocols is listed in below Table-1 on the basis of key features viz. priority consideration, Context awareness and traffic awareness. Priority consideration - A diverse traffic classification exists and urgent traffic is given priority over normal traffic. Context awareness - Contextual information is used to adapt the protocol to various changes in traffic $\&$ channel conditions.

Traffic awareness - Protocol can make traffic adjustments according to network's requirements to avoid wastage of resources

Table 1. Comparison of MAC protocols

\begin{tabular}{|l|c|c|c|}
\hline \multicolumn{1}{|c|}{ Protocol Name } & Priority Consideration & Context Awareness & Traffic Awareness \\
\hline H-MAC [15] & - & - & - \\
\hline U-MAC [2] & $\sqrt{ }$ & - & - \\
\hline BODY-MAC [8] & - & - & - \\
\hline Med-MAC [21] & - & - & $\sqrt{ }$ \\
\hline AR-MAC [1] & - & - & $\sqrt{ }$ \\
\hline A-MAC [19] & - & - & - \\
\hline DQBAN [6] & $\sqrt{ }$ & - & - \\
\hline Body-QoS MAC [28] & $\sqrt{ }$ & - & - \\
\hline BAN MAC [13] & - & - & $\sqrt{ }$ \\
\hline DT-DMA [16] & - & - & $\sqrt{ }$ \\
\hline CA-MAC [5] & - & $\sqrt{ }$ & $\sqrt{ }$ \\
\hline P-MAC [20] & $\sqrt{ }$ & - & $\sqrt{ }$ \\
\hline PLA-MAC [12] & $\sqrt{ }$ & - & $\sqrt{ }$ \\
\hline PNP-MAC [11] & $\sqrt{ }$ & - & $\sqrt{ }$ \\
\hline OCDP [27] & - & $\sqrt{ }$ & $\sqrt{ }$ \\
\hline TAD-MAC [17] & - & - & $\sqrt{ }$ \\
\hline Mc-MAC [18] & $\sqrt{ }$ & - & $\sqrt{ }$ \\
\hline eMc-MAC [26] & $\sqrt{ }$ & - & $\sqrt{ }$ \\
\hline Priority Protocol [4] & & - & \\
\hline
\end{tabular}




\section{Conclusion}

The requirements like low power consumption, scalability, QoS, Reliability, bandwidth utilization make WBAN MAC protocol design a challenging task. In this paper a generic architecture for WBAN was outlined which comprises of sensors, gateway and medical network. Various energy efficient, QoS based, and context aware MAC protocols were described and compared on the basis of their energy efficiency and QoS support. We observed that majority of the reviewed MAC protocols are energy efficient but lack QoS support. Hence there is need to incorporate QoS capabilities into these protocols or entirely new protocols be developed which provide a balance between QoS \& energy efficiency.

\section{References}

[1] A. Rahim, N. Javaid, M. Aslam, U. Qasim and Z. Khan "Adaptive-reliable medium access control protocol for wireless body area networks", IEEE Communications Society Conference on Sensor, Mesh and Ad Hoc Communications and Networks (SECON), (2012), pp. 56-58.

[2] K.A. Ali, J.H. Sarker and Mouftah, "Urgency-based MAC Protocol for Wireless Sensor Body Area Networks", IEEE International Conference on Communications Workshops (ICC), (2010).

[3] A. Thapa and S. Shin, "QoS provisioning in wireless body area networks: A review on MAC aspects", KSII Transactions on Internet and Information Systems, vol. 6, no. 5, (2012), pp. 1267-1285.

[4] B. S. Kim and J. Cho, "A Novel Priority-based Channel Access Algorithm for Contention-based MAC Protocol in WBANs", Proceedings of the 6th International Conference on Ubiquitous Information Management and Communication ICUIMC'12, (2012), Article No.1.

[5] B. Liu and Z. Changwenchen, "MAC Protocol in Wireless Body Area Networks For e-Health: Challenges and a context aware design", IEEE Wireless Communications, (2013), pp. 1536-1284/13.

[6] B. Otal and L. Alonso, "Highly reliable energy saving MAC for wireless body sensor networks in healthcare systems", IEEE journals on selected areas in communication, vol 24, (2009), pp. 553-565.

[7] B. Johny and A. Anpalagan, "Body Area Sensor Networks: requirements, Operations and challenges", IEEE POTENTIALS, vol. 33, (2014), pp. 21-25.

[8] G. Fang and E. Dutkiewicz, "Body MAC: energy efficient TDMA based MAC protocol for wireless body area networks", Proceedings of the 9th International Symposium on Communications and Information Technology (ISCIT), (2009), pp. 1455-1459.

[9] G. V Crosby, T. Ghosh, R. Murimi and C. A. Chin, "Wireless Body Area Networks for Heathcare: A survey", International Journal of Ad hoc, Sensor \& Ubiquitous Computing, (2012), vol. 3, pp. 33013326.

[10] H. Lee, K. Park, B. Lee, J. Choi and R. Elmasri, "Issues in data fusion for healthcare monitoring", Proceedings of the 1st ACM International Conference on Pervasive Technologies Related to Assistive Environments, PETRA, (2008), pp. 86-90.

[11] J.S. Yoon, G.-S. Ahn, S.-S. Joo and M. J. Lee, "PNP-MAC: preemptive slot allocation and nonpreemptive transmission for providing QoS in body area networks", Proceedings of the 7th IEEE conference on Consumer communications and networking conference, (2010), pp. 622-626.

[12] I. Anjum, N. Alam, A. Razzaque, M. Mehedi Hassan and A. Alamri, "Traffic Priority and Load Adaptive MAC Protocol for QoS Provisioning in Body Sensor Networks", International Journal of Distributed Sensor Networks, (2013), Article ID 205192.

[13] K. Shashi Prabh, F. Royo, S. Tennina and T. Olivares, "BANMAC: An Opportunistic MAC Protocol for Reliable Communications in Body Area Networks", IEEE 8th International Conference on Distributed Computing in Sensor Systems (DCOSS), (2012), pp. 166-175.

[14] K. S. Prabh and J.-H. Hauer, "Opportunistic packet scheduling in body area networks", EWSN '11: In Proc. of the 8th European Conference on Wireless Sensor Networks. Springer LNCS, (2011), pp.114129.

[15] L. Huaming and T.Jindong, "heartbeat-driven medium access control for body sensor networks", IEEE transactions on information Technology in Biomedicine, vol. 14, issue 1, (2010), pp. 44-51.

[16] C. Li, H.B. Li and Kohno, "Reservation-based dynamic TDMA protocol for medical body area networks", IEICE TRANSACTIONS on Communications, vol. E92-B, no.2, (2009), pp.387-395.

[17] M. Mahtab Alam, O. Berder, D. Menard and O. Sentieys, "TAD-MAC: Traffic Aware Dynamic MAC Protocol for Wireless Body Area Sensor networks", IEEE JOURNAL ON EMERGING AND SELECTED TOPICS IN CIRCUITS AND SYSTEMS, vol. 2, no. 1, (2012), pp. 109-119.

[18] M. Mostafa Monowar, M. Mehedi Hassan, F. Bajaber, M. Al-Hussein and A. Alamri, "McMAC: Towards a MAC Protocol with Multi-Constrained QoS Provisioning for Diverse Traffic in Wireless Body Area Networks", SENSORS, vol. 12, no. 11, (2012), pp. 15599-15627.

[19] N. Javaid, A. Ahmad, A. Rahim, Z. A. Khan, M. Ishfaq and U. Qasim, "Adaptive Medium Access Control Protocol for Wireless Body Area Networks", International Journal of Distributed Sensor Networks, Article ID 254397, (2014). 
[20] N. Bradai, L. Chaari Fourati, S. Boudjit and L. Kamoun, "New Priority MAC Protocol for Wireless Body Area Networks", ACM MobiHoc Workshop on Pervasive Wireless Healthcare, MobileHealth, (2013), pp. 1-6.

[21] N. F. Timmons and W. G. Scanlon, "An adaptive energy efficient MAC protocol for the medical body area network," 1st International Conference on Wireless Communication, Vehicular Technology, Information Theory and Aerospace and Electronic Systems Technology, Wireless (VITAE '09), (2009), pp. 587-593.

[22] S. Ullah, B. Shen, S.M. Riazul Islam, P. Khan, S. Saleem and K. S. Kwak, "A Study of Medium Access Control Protocols for Wireless Body Area Networks”, Sensors, vol. 10, (2010), pp. 128-145.

[23] S. A. Gopalan and J.-T. Park, "Energy-Efficient MAC Protocols for Wireless Body Area Networks: Survey", International Congress on Ultra-Modern Telecommunications and Control Systems and Workshops (ICUMT), (2010), pp. 739-744.

[24] S. Gonza'lez-Valenzuela, X. Liang, H. Cao, M. Chen and V. C.M. Leung, "Body Area Networks", Springer Series on Chemical Sensors and Biosensors, (2013), pp. 17-38.

[25] S. Hassan and M. Mateen Yaqoob, "Examination of Energy Efficiency for MAC Protocol in Wireless Body Area Networks", International Journal of Computer Applications, vol. 108, no. 20, (2014), pp. 913.

[26] S. Pandit, K. Sarkar, A. Razzaque and A.M. Jehed Sarkar, "An energy efficient multiconstrained QoS aware MAC protocol for body sensor networks", Multimed Tools Appl,, (2015), pp.5353-5374.

[27] Y.-S. Seo, D.-Y. Kim, J. Cho and B. Lee, "OCDP: A WBAN MAC Protocol for Contention-based Medical and CE applications", International Conference on Uniquitous Information Management and Communication ICUIMC, ACM Article No. 12, (2010).

[28] G. Zhou, J. Lu, C.Y. Wan, M.D. Yarvis and J.A. Stankovic, "BodyQoS: Adaptive and Radio-Agnostic QoS for Body Sensor Networks", Conference on Computer Communications on (INFOCOM), (2008), pp. 565-573.

[29] 802.15.6-2012 - IEEE Standard for Local and metropolitan area networks - Part 15.6: Wireless Body Area Networks, vol. 29, (2012). pp. 1-271

[30] IEEE 802.15.4 - (2003), "Wireless medium access control (MAC) and physical Layer (PHY) specifications for low rate wireless personal area networks (LR-WPANs)", IEEE Computer Society, (2003).

[31] C. Li, J. Li, B. Zhen, H. Bang and L.R, Kohn, "Hybrid Unified-Slot Access Protocol for Wireless Body Area Networks", International Journal of Wireless Information Networks, Springer, vol. 17, issue 3, (2010), pp. 150-161.

[32] L. A. Al dulaimi, R. Badlishah Ahmad, L.A. Hassnawi, P. Ehkan and I. Sh. Ahmed, "Performance Comparison of Different MAC Protocols over Wireless Body Area Networks (WBAN)", Australian Journal of Basic and Applied Sciences, (2017), pp. 34-40.

[33] R. Sruthi, "Medium Access Control Protocols for Wireless Body Area Networks: A Survey", Global Colloquium in Recent Advancement and Effectual Researches in Engineering, Science andTechnology (RAEREST) (2016), pp. $621-628$.

[34] K. Khalid and G.-H. Li, "A Survey of MAC Protocols on Wireless Body Area Network", ITM Web of Conferences, DOI: 10.1051/ itmconf/2017203010, (2017).

\section{Authors}

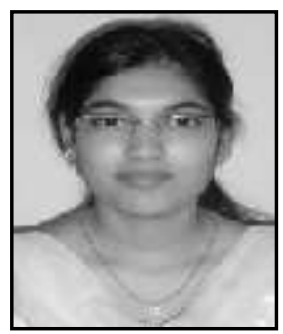

Deepshikha, she is a research scholar at department of computer science and engineering, NIT Hamirpur. Her research interests include Wireless Networks, Body Sensor Networks, Ad Hoc Networks, MAC layer protocols, Routing Protocols, QoS and routing in tactical MANETs.

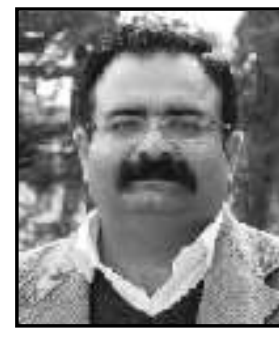

Siddhartha Chauhan, he received the Ph.D. degree in computer science and engineering from National Institute of Technology Hamirpur, H.P., India, in 2013 and the M.Tech. degree in computer science and engineering from Indian Institute of Technology, Roorkee, Uttrakhand, India, in 2003. He has published many research papers in international conferences and journal. $\mathrm{He}$ is currently with Department of Computer Science and Engineering, National Institute of Technology, Hamirpur, H.P., India. His research interests include mobile ad hoc network and wireless sensor network. 
International Journal of Grid and Distributed Computing

Vol. 11, No. 1 (2018) 